\title{
Evaluation of Confocal Fluorescence Spectroscopy for the Detection of Pathological Prion Proteins
}

\author{
Anja Dietrich, Karin Bossart, Bruno Oesch, Christoph Stamm, and Alex J. Raeber
}

\begin{abstract}
Prion diseases or transmissible spongiform encephalopathies (TSEs) are characterized by the accumulation in the brain of $\mathrm{PrPSc}$, an abnormal isoform of the host-encoded glycoprotein $\mathrm{PrPC}^{\mathrm{C}}$. PrPSc is a reliable marker for the post mortem diagnosis of TSEs but its use as a marker for a pre-clinical blood test has been hampered by the low levels of $\mathrm{PrPSc}$ in blood. We have evaluated confocal fluorescence spectroscopy (CFS) as an ultrasensitive method for the immunological detection of PrP in brain homogenates and blood serum. Using recombinant PrP, we determined the detection limit of our CFS assay to be in the picomolar range which is in the same order of magnitude as the corresponding luminescence immunoassay (Prionics ${ }^{\circledR}$-Check LIA). The analytical sensitivity was further investigated using brain homogenates from BSE infected cattle that showed low levels of PrPSc by Western blot analysis. In these brain homogenates, PrPSc was detected by confocal fluorescence spectroscopy down to a dilution of 64-fold whereas the luminescence immunoassay was able to detect PrPSc in brain homogenates diluted down to at least 128 -fold suggesting that in this antibody sandwich assay the luminescence immunoassay has a higher analytical sensitivity than confocal fluorescence spectroscopy. We also demonstrated that blood serum is a suitable matrix for a confocal fluorescence spectroscopy based TSE test, and found that serum did not interfere with the detection of spiked recombinant PrP.
\end{abstract}

Keywords: BSE · Confocal fluorescence scanning microscope · Luminescence immunoassay · Prion protein . TSE diagnosis

\section{Introduction}

Bovine spongiform encephalopathy (BSE) in cattle belongs to the group of transmissible spongiform encephalopathies (TSEs) which also include scrapie in sheep and goats, chronic wasting disease in deer and elk and Creutzfeldt-Jakob disease in human. TSEs are caused by prions which consist of an abnormal form ( $\left.\mathrm{PrP}^{\mathrm{Sc}}\right)$ of the host encod-

${ }^{*}$ Correspondence: Dr. A.J. Raeber

Prionics AG

Wagistrasse 27A

$\mathrm{CH}-8952$ Schlieren

Tel.: +41442002118

Fax: +41442002110

E-Mail: alex.raeber@prionics.ch ed prion protein $\mathrm{PrP}^{\mathrm{C}}$. Prion replication is believed to involve a conformational transition of the mostly $\alpha$-helical $\operatorname{PrP}^{C}$ into the pathological conformer $\mathrm{PrP}^{\mathrm{Sc}}$, which has a high $\beta$-sheet content, aggregates to amyloid fibers and is partly resistant to protease digestion. Prion diseases are characterized by the accumulation of $\mathrm{PrP}^{\mathrm{Sc}}$ in the brains of affected animals and humans. Because $\mathrm{PrPSc}^{\mathrm{Sc}}$ is the only reliable molecular marker for prion diseases, immunological detection of $\mathrm{PrP}^{\mathrm{Sc}}$ in brain tissue forms the basis for post mortem diagnostic tests (for review see [1]).

Since its first recognition in the UK in 1986, BSE has raised great public health concerns because the BSE agent is very likely to cause variant Creutzfeldt-Jakob disease (vCJD) in humans. With the introduction of mandatory active surveillance programs in the European Union the need to develop rapid tests to diagnose BSE has become a high priority. Most rapid diagnostic tests approved by the EU authorities exploit the relative protease resistance of $\mathrm{PrP}^{\mathrm{Sc}}$ in brain samples to discriminate between $\mathrm{PrP}^{\mathrm{C}}$ and $\mathrm{PrP}^{\mathrm{Sc}}$, in combination with immunological detection of the protease resistant part of $\mathrm{PrP}^{\mathrm{Sc}}$ (PrP27-30) [2][3]. In three evaluation campaigns organized by the European Commission (EC) between 1999 and 2004 a total of twelve rapid tests have been approved for BSE monitoring in cattle [4-7]. The twelve rapid tests that are currently used for BSE monitoring in cattle are based on three different techniques for the detection of the pathological form of the prion protein, $\mathrm{PrPSc}^{\mathrm{Sc}}$ : One test is based on Western blot technology [8][9], ten tests are using ELISA technology [10], and in 2004 an immunochromatographic strip test [11] was approved by the EC for BSE testing [6][7].

Currently, $\operatorname{PrP}^{\mathrm{Sc}}$ is used as a marker for the post mortem diagnosis of TSEs. Whether $\mathrm{PrP}^{\mathrm{Sc}}$ can be used as a marker for ante mortem diagnosis of TSEs using blood as a matrix depends on the sensitivity of the detection method because levels of $\mathrm{PrPSc}^{\mathrm{Sc}}$ in peripheral tissues and body fluids are several magnitudes lower than in brain. Infectivity studies in sheep and in rodent models have shown that the levels of prion infectivity in blood are in the order of 1-10 infectious units (IU) $\mathrm{ml}^{-1}$ in the pre-symptomatic period and that these levels rise to about $100 \mathrm{IU} \mathrm{ml}^{-1}$ in the clinical phase [12]. Based on estimations that 100 IU contain 
about 1 picogram of $\operatorname{PrP}^{\mathrm{Sc}}$ [13], it can be assumed that a sensitive blood test would need to detect $\mathrm{PrP}^{\mathrm{Sc}}$ in blood at a level of 10 fg ml $\mathrm{m}^{-1}\left(\sim 3 \times 10^{-16} \mathrm{M}\right)$ to $1 \mathrm{pg} \mathrm{ml}^{-1}(\sim 3 \times$ $\left.10^{-14} \mathrm{M}\right)$. Therefore, highly sensitive methods are a prerequisite for the detection of $\mathrm{PrP}^{\mathrm{Sc}}$ in blood. While current ELISAs are able to detect PrP in the picogram range [10], a number of ultra-sensitive detection methods are being developed with the aim to establish a sensitive diagnostic test for TSEs. Among these techniques are spectroscopy-based methods such as fluorescence correlation spectroscopy (FCS) [14], multispectral ultraviolet fluorescence spectroscopy (MUFS) [15], Fourier-transform infrared spectroscopy (FTIR) [16], and capillary electrophoresis with fluorescent PrP peptides [17].

The confocal microscope was described and patented by Minsky in 1957 [18], and the first detection of a single fluorescently labeled antibody molecule was achieved by Hirschfeld in 1976 [19]. However, these antibodies still contained up to 100 fluoresceine molecules. The detection of a single fluorescent dye molecule was described by Rigler and Widengren in 1990 [20]. With a confocal microscope setup, it is possible to detect a single fluorescent molecule within a femtoliter size volume, corresponding to a nanomolar concentration. Bieschke et al. [14] have used FCS with a confocal microscope for the detection of $\mathrm{PrP}^{\mathrm{Sc}}$ aggregates in solution using antibody probes tagged with fluorescent dyes. With this setup they were able to detect $\mathrm{PrP}^{\mathrm{Sc}}$ in the cerebrospinal fluid of 5 out of 24 CJD patients but in none of the 13 control subjects. Confocal fluorescence spectroscopy (CFS) has been further developed for the ultrasensitive detection of fluorescent molecules on glass surfaces. Loescher et al. [21] described the capture of fluorescently labeled antibodies by a glass surface bound antibody which upon excitation by the laser beam in the confocal detection volume produced single photon bursts over a range of $10^{-10}$ to $10^{-17}$ $\mathrm{M}$ that could be clearly separated from a control sample.

We have developed a method based on CFS using a confocal microscope for the detection of the disease associated form of PrP. By using two anti-PrP specific monoclonal antibodies - a capture antibody immobilized on the glass surface of a microplate and a detection antibody conjugated with a fluorescent dye - we were able to detect recombinant PrP at picomolar concentrations and PrP27-30 in a weakly positive - as determined by Western blot analysis - BSE brain homogenate at dilutions of more than 100-fold. However, a comparison of CFS with a luminescence immunoassay (LIA) using the same antibody sandwich [10] showed similar analytical sensitivities of the two methods.

\section{Materials and Methods}

\subsection{Antibodies and Recombinant Bovine PrP}

The capture and detection antibodies have been described previously [10][22]. Recombinant bovine PrP and the monoclonal antibody (mAb) 6H4 were obtained from Prionics AG (Schlieren, Switzerland). PrP from the same lot (Lot No. 4061778) was used for all experiments and freshly prepared for each experiment by reconstitution of the lyophilized bovine PrP in distilled water.

\subsection{Biotinylation of the Capture Antibody}

Biotinylation of the capture antibody $6 \mathrm{H} 4$ was performed with EZ Link NHSLC-Biotin according to the instructions provided by the manufacturer using an excess of biotin in the reaction (Pierce Biotechnology, Inc., Rockford, IL, USA). The antibody $6 \mathrm{H} 4$ was supplied in $0.2 \mathrm{M}$ carbonate-bicarbonate $\mathrm{pH} \mathrm{9.4,} \mathrm{and} \mathrm{biotin}$ was conjugated via amide bonds in N,N-dimethylformamide. The biotinylation reaction was allowed to proceed for $3 \mathrm{~h}$ with light shaking and unconjugated biotin was then removed by gel filtration on a Sephadex G-25 (Amersham Biosciences Europe $\mathrm{GmbH}$, Freiburg, Germany) column. The conjugation efficiency was determined with the EZ Biotin Quantitation Kit (Pierce Biotechnology, Inc., Rockford, IL, USA) using a bovine serum albumin (BSA)-biotin standard containing eight biotin molecules per BSA. A biotin antibody conjugate with two biotin molecules per antibody was used for the immobilization.

\subsection{Conjugation of the Detection Antibody}

Conjugation of the detection antibody (Prionics AG, Schlieren, Switzerland) with the fluorescent dye $\mathrm{Cy} 5^{\mathrm{TM}}$ was performed as described except that a 4-fold lower amount of Cy5 was used than recommended by the manufacturer. Higher concentrations of Cy5 resulted in an over-conjugation of the antibody and subsequently in a reduction of functional antibody (Amersham Biosciences Europe GmbH, Freiburg, Germany). The antibody was supplied in 0.2 $\mathrm{M}$ carbonate-bicarbonate $\mathrm{pH} 9.4$, and the NHS-activated monofunctional $\mathrm{Cy} 5$ was conjugated to the antibody via amide bonds in N,N-dimethylformamide. The conjugation reaction was performed for 20 min on a rotating wheel in the dark and thereafter unconjugated Cy5 was removed by gel filtration on a Sephadex G-25 column. The conjugation ratio of fluorescent dye $\mathrm{Cy} 5$ to antibody was determined by measuring the absorption between 260 and $750 \mathrm{~nm}$ using an extinction coefficient of 202' $0001 \mathrm{~mol}^{-1}$ $\mathrm{cm}^{-1}$ for the antibody and 250'000 $1 \mathrm{~mol}^{-1}$ $\mathrm{cm}^{-1}$ for Cy5. A conjugation ratio of 3.8 Cy5 molecules per antibody was routinely achieved.

\subsection{Confocal Fluorescence Spectroscopy (CFS)}

Confocal fluorescence measurements were carried out on the LB8 Platereader (Molecular Machines \& Industries, Glattbrugg, Switzerland). The LB8 Platereader incorporates an inverted microscope with an xy-scanning table, a $635 \mathrm{~nm}$ diode laser for excitation, and a single photon avalanche diode for detection. The confocal optics of the microscope brings the light of the laser beam to a tight focus within the well of a microplate which allows detection of single fluorescent molecules in a femtoliter probe volume. Importantly, the use of a small detection area minimizes background signals, which originate either from dye-labeled molecules in solution or from scattered light. Therefore, the read-out is confined to the volume in which the formation of the immunosandwich between PrP and the two antibodies occurs. Glass bottom microplates are mounted on the scanning table, a scan trace of a few millimeters on the bottom of each well is excited by red laser light, and photon bursts from single dye-labeled molecules are collected by the same objective and transmitted to the detector. The photons emitted from single molecules result in peaks distinct from background signals due to scattered light as a result of inhomogeneities on the glass surface. Low concentrations of the analyte can be directly correlated with the number of photons counted in the characteristic peaks produced by single molecules (Fig. 1). However, when high concentrations of the analyte are measured, the peaks produced by single molecules overlap and cannot be analyzed in this way. In this case, all signals above a certain threshold value are integrated and the sum of all photons is recorded. Since these two methods of analysis cannot be combined to a continuous correlation over the whole concentration range of the analyte, we focused on the low concentration range applying the more sensitive quantification of all photons in peaks resulting in a limited dynamic range for the detection of the analyte.

\subsection{Preparation of Capture Antibody Plates}

The LB8 optical paths require the use of glass bottom plates. 384-well glass plates coated by the Langmuir-Blodgett technique [23] with biotinylated cellulose were obtained from Molecular Machines \& Industries (Glattbrugg, Switzerland). The plates were incubated with $1 \times 10^{-8}$ M NeutrAvidin solution (Pierce Biotechnology, Inc., Rockford, IL, USA), washed 


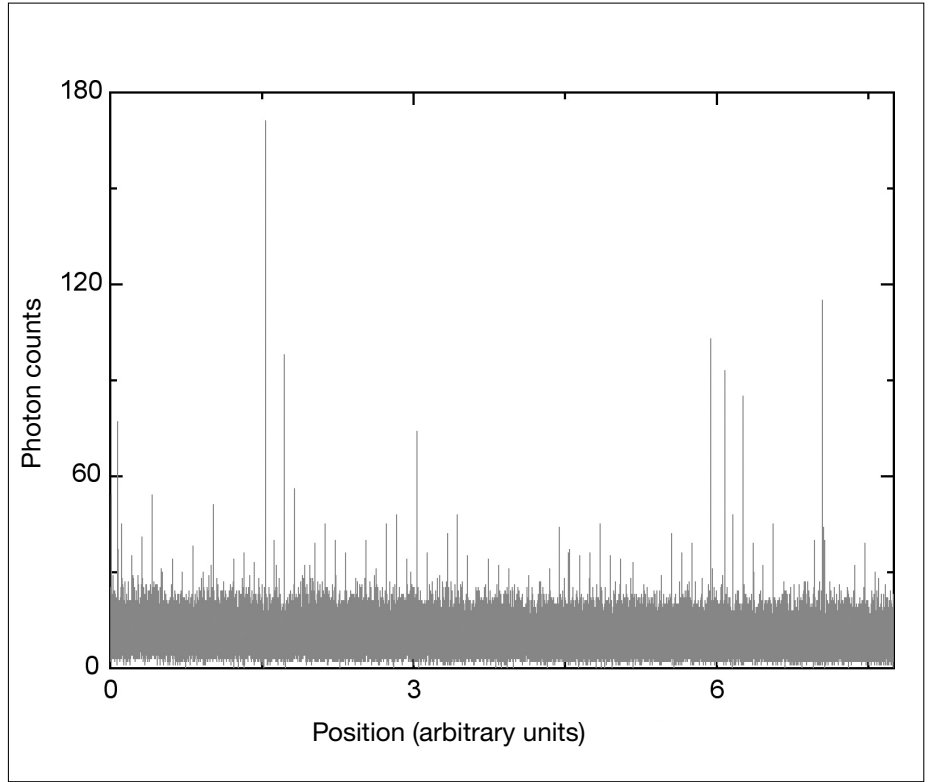

Fig. 1. A typical scan trace monitored by the LB8 Platereader software. The trace is obtained from a scan over a glass surface coated with Cy5 labeled antibodies. Bursts of photons above the background signal of about 30 photons result from single fluorescently labeled antibody molecules bound to the glass surface.

with phosphate buffered saline (PBS) $(0.1$ $\mathrm{M}$ phosphate, $0.15 \mathrm{M} \mathrm{NaCl}, \mathrm{pH}$ 6.9-7.2) then incubated with $1 \times 10^{-8} \mathrm{M} \mathrm{mAb} 6 \mathrm{H} 4-$ biotin solution and washed again with PBS. For the luminescence immunoassay (Prionics ${ }^{\circledR}$-Check LIA) experiments, mAb 6H4 capture plates from the Prionics ${ }^{\circledR}-$ Check LIA kit (Prionics AG, Schlieren, Switzerland) were used.

\subsection{Prionics ${ }^{\circledR}$-Check LIA and LB8 Assay}

The Prionics ${ }^{\circledR}$-Check LIA test was performed as described in the package insert of the test kit. Aliquots of brain homogenates were digested with proteinase K. Recombinant bovine PrP samples and LIA control samples were not treated with proteinase $\mathrm{K}$. Briefly, $15 \mu \mathrm{l}$ of digested brain homogenate or recombinant bovine PrP solution were mixed with $15 \mu$ l assay buffer and 210 $\mu l$ detection antibody in dilution buffer. For the LIA the horse radish peroxidase (POD)antibody conjugate from the kit was used whereas for the LB8 assay an antibody-Cy5 concentration of $1 \times 10^{-10} \mathrm{M}$ was added. Following incubation for $1 \mathrm{~h}$ at room temperature on a shaking platform (500 rpm), $200 \mu \mathrm{l}$ of the reaction mixture were added to the LIA 96-well capture plate, and $80 \mu \mathrm{l}$ were added to the wells of the LB8 384well capture plate. All incubation steps for the LB8 assay were carried out in the dark to prevent photobleaching. After 90 min incubation on a shaking platform (500 rpm), plates were washed and bound antibody was detected in the LIA by addition of the chemiluminescence substrate and the emitted light was measured in a luminom- eter (Microplate Luminometer, Berthold Detection Systems, Pforzheim, Germany). Measurements were performed for one second per well and results are expressed as relative light units (RLU). For fluorescence detection an LB8 Platereader with confocal optics, a $635 \mathrm{~nm}$ diode laser for excitation and a single photon avalanche diode for detection was used in the surface scan mode with a scanned trace of $3 \times 1000 \mu \mathrm{m}$. Data analysis was performed applying peak detection and counting of photons in peaks.

\section{Results}

\subsection{Analytical Sensitivity with Recombinant Bovine PrP}

A method based on confocal fluorescence spectroscopy was evaluated for the detection of the prion protein and compared to the sensitivity of detection by an enzymelinked immunosorbent assay (ELISA). The CFS assay was developed on the LB8 Platereader and was designed as a sandwich immunoassay using the same two anti-PrP antibodies as in a commercially available luminescence immunoassay (Prionics ${ }^{\circledR}$ Check LIA). To compare the sensitivity of PrP detection by CFS with the LIA, we used a series of dilutions of recombinant bovine PrP which were measured in parallel by both technologies.

For the LIA, recombinant bovine PrP was serially diluted from $100 \mathrm{ng} \mathrm{ml}^{-1}$ to $0.39 \mathrm{ng} \mathrm{ml}^{-1}$ and incubated with the horse radish peroxidase (POD) conjugated detection antibody. The PrP-antibody complex was then transferred to a $6 \mathrm{H} 4$-coated cap- ture plate. After washing, chemiluminescence substrate was added and light emission measured in a luminometer. The values of five measurements were used to calculate the mean and standard deviation for each PrP concentration and are expressed as relative light units (RLU). The limit of detection of the LIA was determined at a PrP concentration of $780 \mathrm{pg} \mathrm{ml}^{-1}$ which corresponds to a final concentration in the well of the microplate of $49 \mathrm{pg} \mathrm{ml}^{-1}$ or $\sim 2$ $\times 10^{-12} \mathrm{M}$ using the molecular weight of 23' $690 \mathrm{~g} \mathrm{~mol}^{-1}$ for recombinant bovine PrP (Fig. 2A). Even at the highest PrP concentration of $100 \mathrm{ng} \mathrm{ml}^{-1}$ there was no signal saturation observed demonstrating the wide dynamic range of the LIA of more than three orders of magnitude [10]. The background was found to be due to unspecific binding of the detection antibody to the 6H4-coated capture plate since the signal with $\operatorname{PrP}$ alone ('no det $A b$ ') was about 10-fold lower than the signal with the detection antibody alone ('no PrP'). Reproducibility of the LIA assay was excellent as evidenced by the small standard deviations $(<10 \%)$.

The same samples used in the LIA assay were analyzed in parallel with the CFS technology. Recombinant PrP was incubated with $1 \times 10^{-10} \mathrm{M}$ Cy5-conjugated detection antibody, the reaction mixture was transferred to a 384-well glass plate coated with biotinylated cellulose, NeutrAvidin and biotinylated $6 \mathrm{H} 4$ antibody and fluorescent targets on the plate surface were detected by scanning the surface with the laser. From the different options of analysis, counting of photons in peaks was determined to be the most sensitive method. The peak threshold was chosen according to the background signal of the blank samples, and the width of all collected peaks was smaller than 5 bins to exclude unwanted signals ( 1 bin corresponds to $1 \mu \mathrm{m}$ in the lateral dimension). Each PrP dilution was analyzed fivefold to investigate well-to-well variations and to calculate mean values and standard deviations. A detection limit of $3.13 \mathrm{ng} \mathrm{ml}^{-1}$ was determined for the LB8 assay which corresponds to a final well concentration of 196 pg ml ${ }^{-1}$ or $\sim 8 \times 10^{-12} \mathrm{M}$ (Fig. 2B) showing that the detection limits of both the LIA and CFS are in the same order of magnitude. However, the LB8 technology has a lower dynamic range than the LIA as shown by the decrease in the number of photons in peaks at concentrations higher than $50 \mathrm{ng} \mathrm{ml}^{-1}$. At these high concentrations single peaks can no longer be detected. Similar to the LIA, the detection antibody gave some unspecific binding to the $6 \mathrm{H} 4$-coated capture plate - compare signals with detection antibody alone ('no PrP') with the signals with PrP alone ('no det $\mathrm{Ab}$ '). No peaks from background fluorescence were detected when both PrP and the detection antibody were omitted. Reproducibility of the LB8 assay 

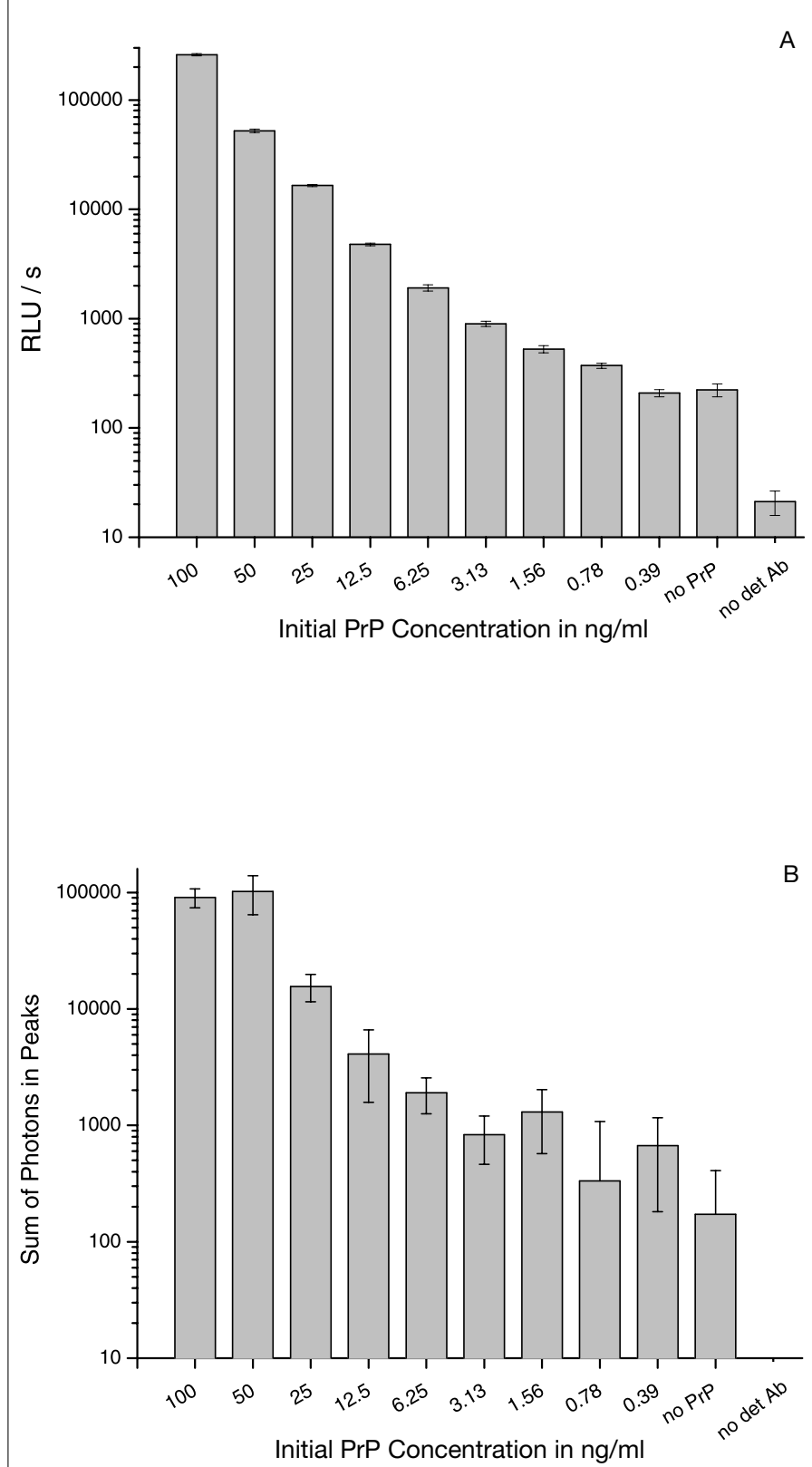

Fig. 2. Analytical sensitivity of the Prionics ${ }^{\circledR}$-Check LIA assay (A) and the LB8 assay (B) with recombinant bovine PrP. Different amounts of recombinant bovine PrP were measured with the two assays omitting the proteinase $\mathrm{K}$ digestion step because recombinant PrP is not proteaseresistant. RLU s s${ }^{-1}$ values obtained from the Prionics ${ }^{\circledR}-$ Check LIA or the sum of photons in peaks from the LB8 assay are logarithmically plotted against the concentrations of recombinant PrP ranging from $100 \mathrm{ng} \mathrm{ml}^{-1}$ to $390 \mathrm{pg} \mathrm{ml}^{-1}$. As controls either $\operatorname{PrP}$ (no PrP) or the detection antibody (no $\operatorname{det} A b)$ were omitted from the reaction.

was clearly inferior to the LIA as shown by the high standard deviations of $44 \%$ without even including the outlier at a dilution of $0.78 \mathrm{ng} \mathrm{ml}^{-1}$.

\subsection{Analytical Sensitivity with BSE Brain Homogenates}

We next investigated the analytical sensitivity of the two assays for the detection of the misfolded form of the prion protein $(\mathrm{PrP})$ in BSE infected cattle brain homogenates using protease digestion to distin- guish $\mathrm{PrP}^{\mathrm{Sc}}$ from its cellular counterpart $\mathrm{PrP}^{\mathrm{C}}$. A tissue sample from the obex region of a Western blot confirmed BSE positive cow with low levels of $\mathrm{PrPSc}^{\mathrm{Sc}}$ was homogenized, the brain homogenate was serially diluted up to 256-fold into BSE negative brain homogenate and then digested with proteinase K. For the LIA assay, PrP27-30 in the digested brain homogenate was incubated with the HRP conjugated detection antibody, the PrP-antibody complex was then transferred to a $6 \mathrm{H} 4$-coated capture plate, and the generated RLUs ${ }^{-1}$ of five measurements were used to calculate the mean and standard deviation for each dilution. Within this dilution range the limit of detection was determined to be between the dilutions of 1:128 to 1:256 (Fig. 3A). Similar to the recombinant PrP samples, the LIA showed no signal saturation with the higher concentrated brain samples. A BSE negative homogenate produced a 5.5fold higher signal than the water control ('no hom'). The standard deviations were slightly higher than for recombinant PrP, and the average error for the BSE positive brain homogenates was $24 \%$.

Aliquots of the digested BSE brain homogenates were analyzed in parallel with the LB8 assay. Results of five measurements were used to calculate the mean and standard deviation for each dilution. Peak analysis and determination of the sum of photons in all peaks resulted in the detection of dilutions up to 1:64 (Fig. 3B). Higher dilutions showed more signal variations and were not significantly different from the background signal. The higher concentrated samples did not yield any peaks showing again the lower dynamic range of the LB8 technology considering that data analysis was optimized for the low concentration range of the analyte. The standard deviations of the 1:16 to $1: 256$ diluted samples gave an average error of $17 \%$. The 1:8 dilution was not included in this analysis because the $\mathrm{PrP}^{\mathrm{Sc}}$ concentration was too high for the application of peak detection.

\subsection{Analytical Sensitivity with Recombinant PrP Spiked into Blood Serum}

In TSEs, prions and $\mathrm{PrPSc}^{\mathrm{Sc}}$ replicate efficiently in the central nervous system and, hence, brain tissue is used as the target organ for the post mortem diagnosis using rapid tests. However, a routine pre-symptomatic test for TSEs would require examination of easily accessible body fluids such as urine, saliva or blood. Although, the presence of $\mathrm{PrP}^{\mathrm{Sc}}$-like molecules has been reported in urine of TSE infected animals and humans [24], this report has been questioned by results showing cross-reactivity of anti-PrP antibodies with bacterial proteins present in urine [25]. Because prions were found in the blood of scrapie infected sheep [12], blood seems the best option as a matrix for a non-invasive TSE test for live animals and humans. To evaluate blood as a matrix for the detection of PrP, we performed spiking experiments of recombinant bovine $\mathrm{PrP}$ into bovine blood serum.

For the LIA, recombinant bovine $\mathrm{PrP}$ was serially diluted in serum from $20 \mathrm{ng}$ $\mathrm{ml}^{-1}$ to $0.4 \mathrm{ng} \mathrm{ml}^{-1}$ and incubated with the HRP conjugated detection antibody. The PrP-antibody complex was then transferred to a 6H4-coated capture plate and sig- 


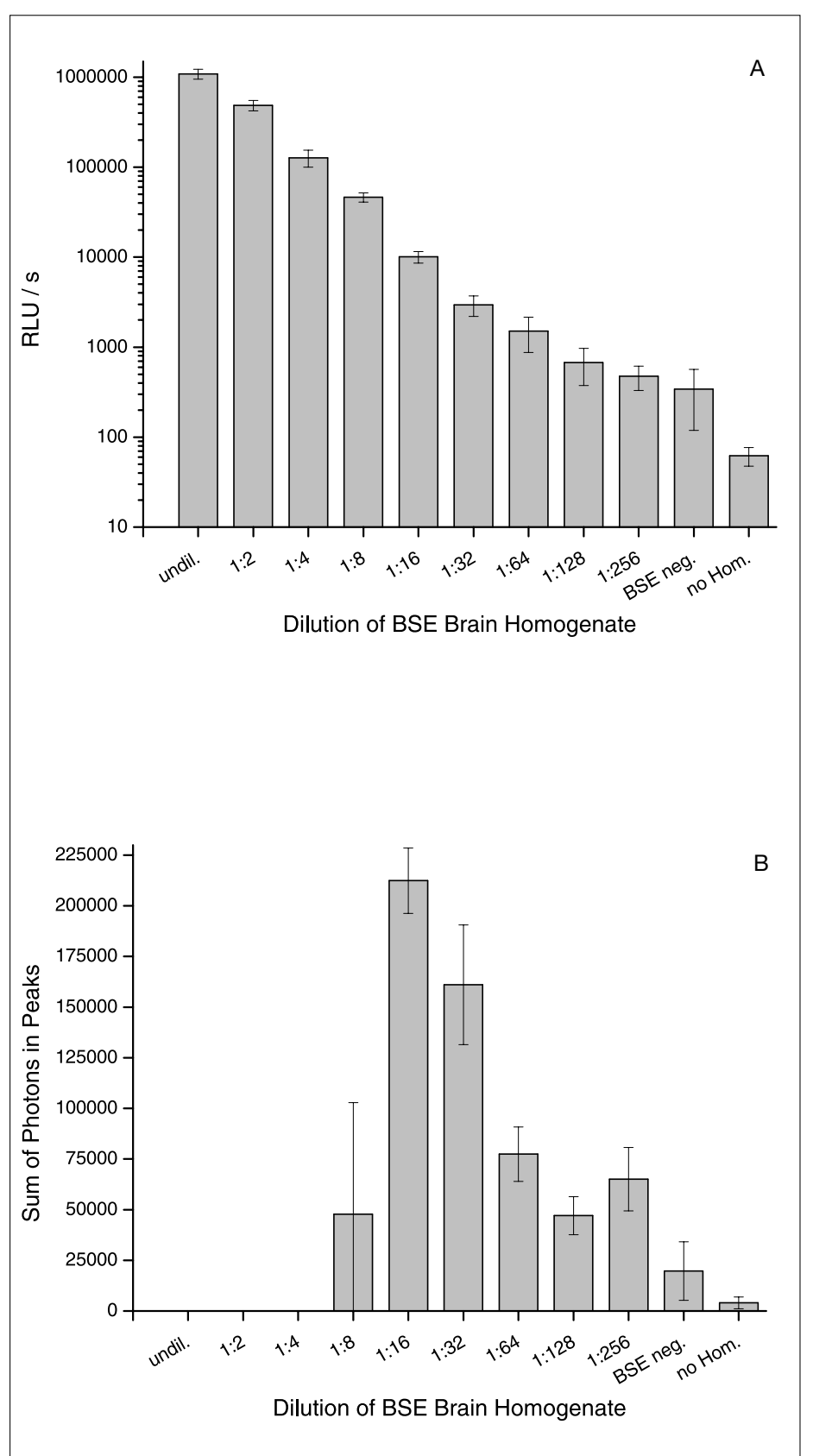

Fig. 3. Analytical sensitivity of the Prionics ${ }^{\circledR}$-Check LIA assay (A) and the LB8 assay (B) with BSE brain homogenates. A BSE positive brain homogenate was serially diluted into a negative brain homogenate. The samples were digested with proteinase $\mathrm{K}$ and PrP27-30 was measured with the two assays. RLU s ${ }^{-1}$ values obtained from the Prionics ${ }^{\circledR}$-Check LIA or the sum of photons in peaks from the LB8 assay are logarithmically plotted against the dilutions ranging from 1:2 to 1:256. As controls, either a BSE negative brain homogenate (BSE neg) or PBS (no Hom.) were used in the reaction.
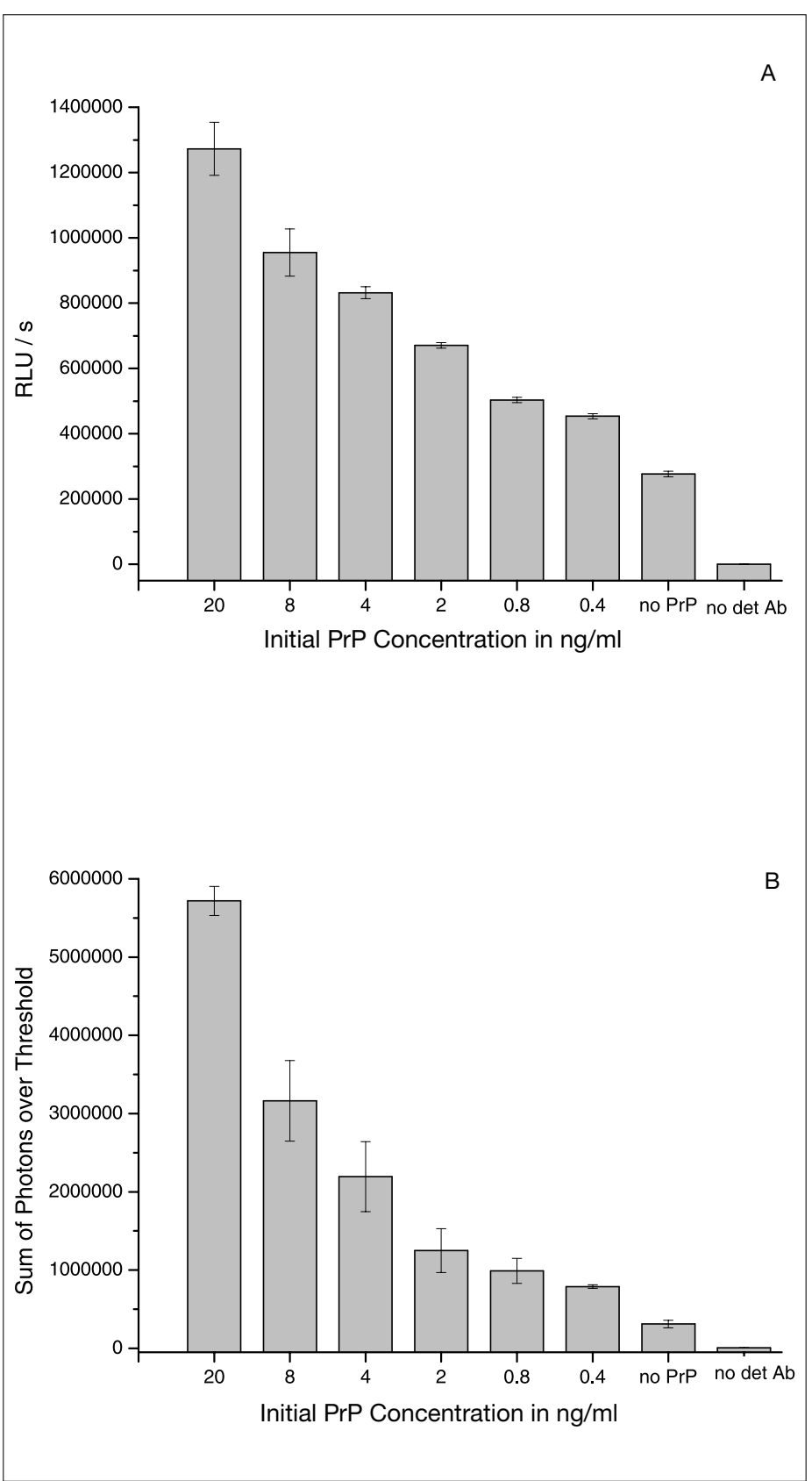

Fig. 4. Analytical sensitivity of the Prionics ${ }^{\circledR}$-Check LIA assay (A) and the LB8 assay (B) with recombinant PrP spiked into blood serum. Recombinant bovine PrP was serially diluted into bovine blood serum and measured with the two assays. RLU s ${ }^{-1}$ values obtained from the Prionics ${ }^{\circledR}$-Check LIA or the sum of photons in peaks from the LB8 assay are plotted against the concentrations of recombinant PrP ranging from $20 \mathrm{ng} / \mathrm{ml}$ to $400 \mathrm{pg} / \mathrm{ml}$. As controls either PrP (no PrP) or the detection antibody (no det Ab) were omitted from the reaction. nal detection was performed as described above. For the LB8 assay, aliquots of the same PrP dilutions were incubated with Cy5-conjugated detection antibody, transferred to the 6H4-coated 384-well capture plate and the signals were analyzed with the LB8 Platereader. In this experiment a scan sensitivity of $10 \times 1000 \mu \mathrm{m}$ was used and data were analyzed by collecting all photons above background threshold. Peak analysis was not performed in this experiment to cover a higher dynamic range. In both assays, LIA and LB8, blood serum as a matrix did not cause interference with the read-out nor did it affect significantly the intrinsic background as shown in the reaction without adding the detection antibody conjugate ('no det $\mathrm{Ab}$ ') (Fig. 4). The detection limit for recombinant PrP spiked into serum was found to be at least $400 \mathrm{pg} \mathrm{ml}^{-1}$ which corresponds to a final concentration of about $50 \mathrm{pg} \mathrm{ml}^{-1}$ or $\sim 2 \times 10^{-12} \mathrm{M} \operatorname{PrP}$ in the well of the microplate. Since this detection limit is in the same order of magnitude as with PrP diluted in buffer (Fig. 2), we conclude that serum does not show a sig- nificant interference with the detection of PrP in the two assay platforms. Serum alone without spike showed a significant signal in both assays which is due to endogenous $\mathrm{PrP}^{\mathrm{C}}$ in serum as well as unspecific binding of the detection antibody.

\section{Discussion}

Recently, the first cases of possible blood transfusion transmitted vCJD have been reported in the UK [26][27]. If the pos- 
sibility of transmission of vCJD by blood transfusion is supported by the discovery of similar cases in the future, this could have serious implications for a vCJD epidemic and underscores the importance of developing tests that are capable of detecting prions in blood to assure the safety of the human blood supply.

To date, $\mathrm{PrP}^{\mathrm{Sc}}$ is used as a marker for the post mortem diagnosis of TSEs because high concentrations are found in the CNS of infected animals and humans. In contrast, $\mathrm{PrP}^{\mathrm{Sc}}$ concentrations in blood are extremely low, if present at all, and ultra-sensitive methods to detect PrP become mandatory for ante mortem test development. Although progress has been made recently to develop highly sensitive detection methods for serological assays, only a few reports have been published regarding the use of such methods for the detection of the pathological form of PrP.

We have developed an immunological assay based on confocal fluorescence spectroscopy for the detection of PrP. This assay was evaluated with regard to sensitivity for the detection of $\mathrm{PrP}^{\mathrm{Sc}}$ in $\mathrm{BSE}$ affected cattle brain homogenates as well as for recombinant PrP spiked into serum as a model for a blood-based TSE test. Both assay technologies investigated in this report, the LIA and the LB8 test, use the same sample preparation and antibodies for the detection of PrP. Contrary to our expectations, CFS showed a similar analytical sensitivity to the LIA for recombinant PrP as well as for diluted BSE brain homogenates. Further, reproducibility was clearly better for the LIA than for CFS as evidenced by the high variation of signals in the LB8 assay. This experimental variation is most likely due to insufficient homogeneity in the coating of the plate as a result of the manufacturing process and could represent a serious constraint for a routine diagnostic assay.

While the LIA had a wide dynamic range covering more than three orders of magnitude, the LB8 assay showed limitations in the detection of higher concentrations of the analyte. At higher concentrations, peak analysis cannot be applied because single peaks cannot be resolved resulting in a decrease in the peak count. Therefore, at higher concentrations, overall counting of photons was employed and, hence, interfering signals cannot be excluded from the calculation. Another disadvantage of the LB8 technology is that the background value has to be defined for each data set, and it is also more difficult to define a cutoff value in the LB8 assay than for the LIA. Overall, the LB8 technology does not show a higher analytical sensitivity than the LIA, even when the path of the scan trace (and therefore scan time) is extended. However, the sensitivity of the LB8 technology could possibly be further optimized for PrP de- tection by changing parameters such as the density and nature of the plate coating or by using direct coating of the antibody to the glass surface, co-immobilization of blocking molecules, changing buffers to reduce unspecific binding of detection antibodies, or even using alternative antibody sandwiches. In summary, CFS has potential as a tool in ante mortem TSE diagnostics but further optimization is necessary to achieve the sensitivities required for the detection of $\mathrm{PrP}^{\mathrm{Sc}}$ in blood of humans and animals affected with TSEs.

\section{Acknowledgements}

We would like to thank Stefan Niehren, Frank Loescher, Stefan Jung, and Stefan Seeger from Molecular Machines \& Industries for scientific support, Langmuir-Blodgett coating of plates, and technical advice.

Received: April 15, 2005

[1] C. Weissmann, Nat. Rev. Microbiol. 2004, 2,861 .

[2] B. Oesch, D. Westaway, M. Walchli, M.P. McKinley, S.B. Kent, R. Aebersold, R.A. Barry, P. Tempst, D.B. Teplow, L.E. Hood, S.B. Prusiner, C. Weissmann, Cell 1985 40, 735.

[3] D.C. Bolton, M.P. McKinley, S.B. Prusiner, Science 1982, 218, 1309.

[4] J. Moynagh, H. Schimmel, G.N. Kramer, http://europa.eu.int/comm/food/fs/bse/ bse12_en.html, 1999.

[5] H. Schimmel, P. Catalani, L. Le Guern, G.N. J. Prokisch, W. Philipp, S. Trapmann, R. Zeleny, J. Moynagh, http://europa.eu.int/comm/food/food/biosafety/bse/ bse42_en.pdf, 2002.

[6] W. Philipp W, P. van Iwaarden, M. Goll, N. Kollmorgen, H. Schimmel, P. Vodrazka, http://www.irmm.jrc.be, 2004.

[7] Anonymous, http://www.efsa.eu.int/science/ tse assessments/bse tse/694_en.html, 2004.

[8] O. Schaller, R. Fatzer, M. Stack, J. Clark, W. Cooley, K. Biffiger, S. Egli, M. Doherr, M. Vandevelde, D. Heim, B. Oesch, M. Moser, Acta Neuropathol. (Berl.) 1999, 98, 437.

[9] B. Oesch, M. Doherr, D. Heim, K. Fischer S. Egli, S. Bolliger, K. Biffiger, O. Schaller, M. Vandevelde, M. Moser, Arch. Virol. Suppl. 2000, 16, 189.

[10] K. Biffiger, D. Zwald, L. Kaufmann, A Briner, I. Nayki, M. Pürro, S. Böttcher, T. Struckmeyer, O. Schaller, R. Meyer, R. Fatzer, A. Zurbriggen, M. Stack, M. Moser, B. Oesch, E. Kübler, J. Virol. Methods 2002, 101, 79

[11] E. Kuebler, B. Oesch, P. Price, WO Patent No. 2003029813, 2003

[12] N. Hunter, J. Foster, A. Chong, S. McCutcheon, D. Parnham, S. Eaton, C. MacKenzie, F. Houston, J. Gen. Virol. 2002, 83, 2897.

[13] P. Brown, L. Cervenakova, H. Diringer, J. Lab. Clin. Med. 2001, 137, 5.
[14] J. Bieschke, A. Giese, W. Schulz-Schaeffer, I. Zerr, S. Poser, M. Eigen, H. Kretzschmar, Proc. Natl. Acad. Sci. USA 2000, 97, 5468 .

[15] R. Rubenstein, P.C. Gray, C.M. Wehlburg, J.S. Wagner, G.C. Tisone, Biochem. Biophys. Res. Commun. 1998, 246, 100.

[16] P. Lasch, J. Schmitt, M. Beekes, T. Udelhoven, M. Eiden, H. Fabian, W. Petrich, D. Naumann, Anal. Chem. 2003, 75, 6673.

[17] M.J. Schmerr, A.L. Jenny, M.S. Bulgin, J.M. Miller, A.N. Hamir, R.C. Cutlip, K.R. Goodwin, J. Chromatogr. 1999, 853, 207.

[18] M. Minsky, Microscopy apparatus, US 3013467, 1969.

[19] T. Hirschfeld, Appl. Opt. 1976, 15, 2965.

[20] R. Rigler, J. Widengren, Bioscience 1990, 3,180 .

[21] F. Loescher, S. Bohme, J. Martin, S. Seeger, Anal. Chem. 1998, 70, 3202.

[22] C. Korth, B. Stierli, P. Streit, M. Moser, O. Schaller, R. Fischer, W. Schulz-Schaeffer, H. Kretzschmar, A. Raeber, U. Braun, F. Ehrensperger, S. Hornemann, R. Glockshuber, R. Riek, M. Billeter, K. Wuthrich, B. Oesch, Nature 1997, 390, 74.

[23] F. Loescher, S. Seeger (Molecular Machines \& Industries, GmbH), DE 59803449D, 2002.

[24] G.M. Shaked, Y. Shaked, Z. Kariv-Inbal, M. Halimi, I. Avraham, R. Gabizon, J. Biol. Chem. 2001, 276, 31479.

[25] H. Furukawa, K. Doh-ura, R. Okuwaki, S. Shirabe, K. Yamamoto, H. Udono, T. Ito, S. Katamine, M. Niwa, J. Biol. Chem. 2004, 279, 23661

[26] C.A. Llewelyn, P.E. Hewitt, R.S. Knight, K. Amar, S. Cousens, J. Mackenzie, R.G. Will, Lancet 2004, 363, 417.

[27] A.H. Peden, M.W. Head, D.L. Ritchie, J.E. Bell, J.W. Ironside, Lancet 2004, 364, 527. 